\title{
Höhere Anlagenperformance in der Prozessindustrie
}

\author{
Performancekennzahlen zur Entscheidungsunterstützung
}

\begin{abstract}
Der Beitrag zeigt systematisch auf, wie sich Maßzahlen zur Bewertung der Anlagenperformance in der Prozessindustrie nutzen lassen. Dabei wird der Fokus auf die operative, zeitnahe Nutzung der Maßzahlen gelegt. Es wird Bezug auf Forschungsergebnisse des EU-Projektes MORE genommen. Industriell umgesetzte Beispiele verdeutlichen den Nutzen, aber ebenso die Herausforderungen bei der Umsetzung von Lösungen zur Steigerung der Anlagenperformance durch Maßnahmen im operativen Betrieb.
\end{abstract}

\section{SCHLAGWÖRTER Performancekennzahlen / KPI / Anlagenperformance /} Informationsmodellierung / Betriebsassistenz

\begin{abstract}
Improving plant performance in the process industry - Using indicators to analyse performance

The paper illustrates how performance indicators can be used to analyse and improve plant performance, focusing on the use of indicators in real-time to support the operational decisions in the day-to-day operations of chemical production processes. The contributions are based mainly on results of the EU project MORE. In addition, industrial applications are considered and challenges to implementing solutions in practice are discussed. Finally, current research activities are outlined and an overview is given of possible future applications.
\end{abstract}

KEYWORDS plant performance / performance indicators / information modelling / production assistance 


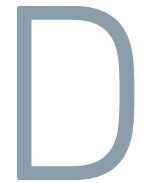

er Bedarf Produktionsanlagen bestmöglich zu betreiben, ist eine Motivationsquelle, die als Selbstverständlichkeit angesehen und seit jeher verfolgt wird. Das Verständnis zur Beurteilung eines bestmöglichen Betriebs und damit zur Beurteilung einer Anlagenperformance unterliegt jedoch einem stetigen Wandel. Die Fragen der Messbarkeit einer Anlagenperformance und der Eignung von Indikatoren zur eindeutigen Ursache-Wirkungsanalyse und darauf aufbauend zur Ableitung von Maßnahmen zur Verbesserung der Performance müssen stetig überdacht und neu beantwortet werden. Die Entwicklung und Nutzung geeigneter Maßzahlen kann entscheidend dazu beitragen, ob Effizienzmanagementsysteme und Systeme zur Entscheidungsunterstützung einen nachhaltigen Nutzen aufweisen, siehe unter anderem [1-3].

Die Herausforderung besteht zum einen darin, dass die Beurteilung der Anlagenperformance mehreren Teilzielen unterliegt, die sich teilweise gegenseitig beeinflussen und sich nicht selten durch Prozessführungsänderungen konträr zueinander verändern können. Zum anderen ist es zur Bewertung der Anlagenperformance immer wichtig, geeignete Bezugspunkte zur Beurteilung von Verbesserungs- oder Verschlechterungstendenzen zu finden. Bei ungeeigneten Indikatoren oder Bezugspunkten kann es im schlimmsten Fall passieren, dass Effizienzverbesserungen angezeigt werden, obwohl eine Verschlechterung eingetreten ist.

Im Bereich der Energieeffizienz bekommt dieser Aspekt eine besondere Bedeutung, da bei Audits gemäß ISO 50001 [4] beziehungsweise ISO 50003 [5] ab 2017 erstmals messbare Steigerungen der Energieeffizienz nachgewiesen werden müssen, um an steuerlichen Vergünstigungen partizipieren zu können.

\section{WANDEL DER PERFORMANCEBEURTEILUNG}

Das Verständnis für den Begriff der Anlagenperformance hat sich in den vergangenen Jahren weiterentwickelt, siehe Bild 1. Mit der Einführung von Prozessleitsystemen und den Methoden der Regelungstechnik stand zunächst die Steigerung der Prozessstabilität und der Robustheit im Fokus. Unterstützt durch Betriebsleitsysteme (MES) sowie Simulationswerkzeuge wurde dann vermehrt die Verbesserung der Produktqualität und des Durchsatzes angestrebt.

Insbesondere ab den 90er-Jahren wurde versucht, Forderungen nach Steigerungen der Anlagenverfügbarkeit zu erfüllen. Zum Einsatz kamen dabei Lösungen des Plant Asset Managements und des Online Condition Monitorings. In den letzten Jahren wurde vermehrt die Energie- und Materialeffizienz betrachtet. Neuartige Messtechniken inklusive Onlineprozessanalysen helfen dabei, hier einen sukzessiven Fortschritt zu erzielen.

Aktuell und vermutlich noch intensiver in naher Zukunft wird zunehmend das flexible Betreiben der Anlagen nach aktuellem Marktbedarf und aktuellen Energie- und Rohstoffpreisen gefordert. Grund dafür ist die erhöhte Dynamik der Einkaufs- und Verkaufsbedingungen. Die gestiegene Dynamik des Marktbedarfs ist heute ein wesentlicher Treiber, um die Wertschöpfungsketten weiter zu optimieren. Der Bedarf nach kleineren Losgrößen, kürzere Time to Market

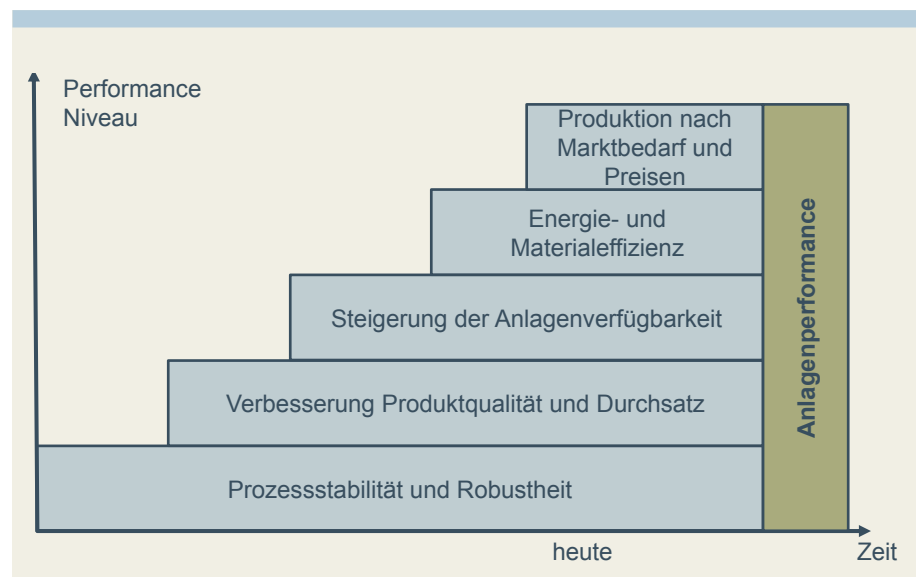

BILD 1: Kriterien der Anlagenperformance 


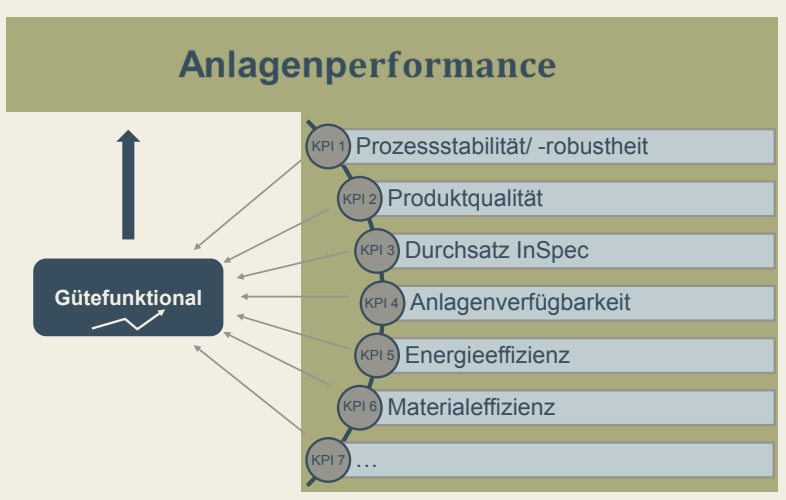

BILD 2: Bildung von Gütefunktionalen

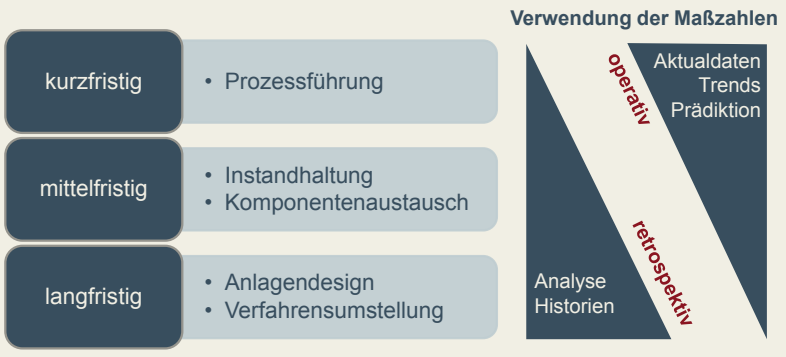

BILD 3: Operative und retrospektive Nutzung von Maßzahlen

- Anforderungen neuer Produkte und die daraus resultierenden Ansprüche an die Flexibilität der Anlagen bedürfen neuer Herangehensweisen und Ansätze zur Steigerung der Anlagenperformance mit Mitteln produktionsnaher IT-Lösungen, die im operativen Betrieb eingesetzt werden.

Alle Kriterien zusammen bestimmen heute die Anlagenperformance. Die Anlagenperformance stellt damit einen Begriff dar, der den Nutzen und die Effizienz im Sinne einer Bewertung des Nutzens im Verhältnis zum erforderlichen Einsatz - einer Anlage wiedergibt. Die Performance setzt sich dabei meist aus einer Kombination von Aspekten zusammen, was dann in einer multikriteriellen Bewertung resultiert.

Zur quantitativen Ermittlung einer Aussage zur Anlagenperformance müssen den einzelnen Bewertungsaspekten zunächst Maßzahlen zugeordnet werden. Man spricht von Performance Indicators (PI) und bei besonders wichtigen Bewertungsgrößen von Key Performance Indicators (KPI). Allein die Definition, Erfassung und Visualisierung beziehungsweise Reporting einer Menge an unabhängigen KPI stellt bereits einen nicht zu unterschätzenden Nutzen dar, um kontinuierlich geeignete Maßnahmen zur Verbesserung der Anlageneffizienz abzuleiten.
Es ist in der Regel nicht möglich, alle Ziele gemeinsam zu optimieren. Daher bedarf es für eine zentrale Aussage zur aktuellen Anlagenperformance der Formulierung eines Gütefunktionals, siehe Bild 2. Dieses Gütefunktional kann mathematisch beschrieben werden, zum Beispiel in dem die einzelnen KPI als Teilziele aufgefasst, gewichtet und dann zu einer gemeinsamen Zielfunktion zusammengefasst werden. Oftmals wird das Gütefunktional aber implizit - basierend auf Erfahrungswerten oder durch die Reduktion auf eine oder wenige Zielgrößen - in den Köpfen der Entscheider gebildet.

Erschwerend kommt hinzu, dass das Gütefunktional selber in der Regel dynamisch ist. Bei Vollauslastung der Anlagen kann es vorkommen, dass aus wirtschaftlicher Betrachtung der Anlagenverfügbarkeit und dem Durchsatz ein höherer Performancebeitrag zugemessen wird als beispielsweise der Energie- oder Materialeffizienz. In Zeiten geringerer Nachfrage oder bedingt durch andere Faktoren können dagegen die Energieoder Materialeffizienz bei der Bewertung der Plant Performance wieder eine höhere Gewichtung bekommen.

\section{MAßZAHLEN}

Maßzahlen zur Bestimmung einer Anlagenperformance werden in diversen Normen und Richtlinien definiert. Dazu zählen insbesondere die internationalen Normen ISO 22400 [6] und ISO/IEC 20140 [7] sowie die nationalen Richtlinien VDMA 66412 [8] und VDI 4800 [9]. Zur Bestimmung von Energie- und Materialeffizienzkenndaten, die zur Entscheidungsunterstützung und Optimierung im operativen Betrieb genutzt werden können, wird 2017 die Namur-Empfehlung NE 162 Ressourceneffizienzindikatoren für die Überwachung und Verbesserung der Ressourceneffizienz in verfahrenstechnischen Anlagen [10] erscheinen, die zur Zeit in einer Joint Working Group des Namur-AK 4.17 Energieeffizienz und des AK 2.4 MES erarbeitet wird und auf Vorarbeiten des EU-Forschungsprojektes MORE [11] basiert. Vorgehensmodelle zur Nutzung von Ressourceneffizienzindikatoren, die auch verallgemeinerbar bei der Umsetzung anderer Performanceaspekte genutzt werden können, werden in [12] und [13] sowie, speziell für diskontinuierlich betriebene Anlagen, in [14] beschrieben. Eine ausführliche Darstellung und Diskussion wird in [15] erscheinen.

Auf Basis von Maßzahlen können Verbesserungsmaßnahmen abgeleitet werden. Die Stellhebel zur Verbesserung der Anlagenperformance sind:

- langfristig: Änderungen des Anlagendesigns oder Verfahrensumstellungen,

- mittelfristig: Umstellung von Instandhaltungsstrategien oder Komponententausch,

- kurzfristig: Variation der Prozessführung.

Die Maßzahlen können dabei sowohl retrospektiv also auf Basis der Historien - als auch operativ genutzt werden. Zur Ableitung der langfristigen Maßnahmen 


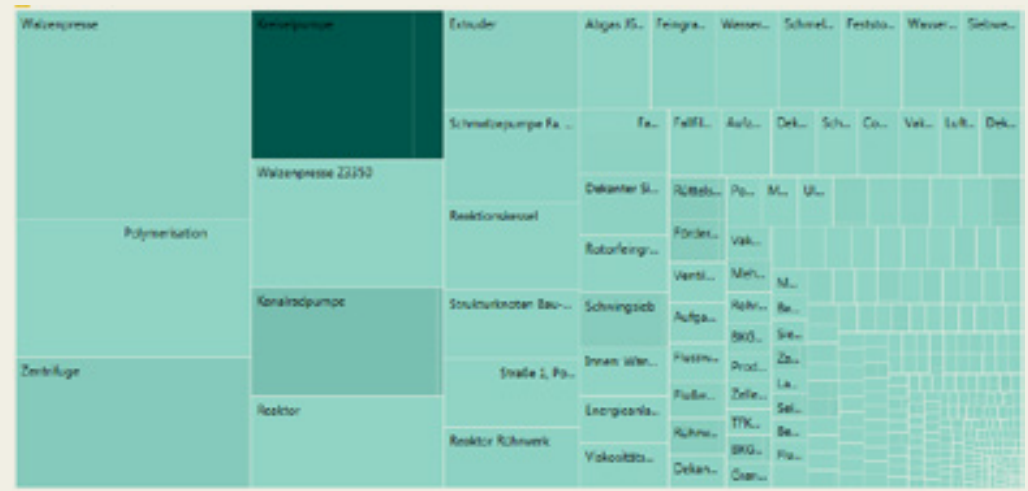

a) Treemap

b) Word-Cloud

BILD 4: Instandhaltungsanalysen

überwiegt die retrospektive Betrachtung, für die kurzfristigen, operativen Strategien werden vermehrt die Aktualdaten, zeitlich naheliegende Historien und vermehrt auch Prädiktionen verwendet, siehe Bild 3.

Bei der retrospektiven Nutzung der Plant Performance KPI wird die Historie der Maßzahlen betrachtet. Ziel ist es, Verbesserungspotenziale und Performancetendenzen zu erkennen. Neben grundsätzlichen Entscheidungen zum Anlagendesign und der Fahrweisenoptimierung können dabei Analysen und Optimierungen, zum Beispiel für Reinigungs- und Instandhaltungszyklen oder auch für Anlagenbelegungen und Auftragsmanagement im Rahmen der Grobplanung, genutzt werden. Systemtechnische Funktionen, die dazu benötigt werden, sind unter anderem statistische Analysen und Reports. Durch diese Analysen lassen sich Schwachstellen und Probleme, insbesondere systematische Probleme und Fehler einer Anlage, erkennen.

Ein Beispiel eines Reports ist eine Bad-Actor-Analyse, die im Rahmen des Instandhaltungsmanagements eingesetzt werden kann. Unterschiedliche instandhaltungsrelevante Anlagenkomponenten werden in Form einer Treemap aufgeführt, siehe Bild 4. Hier stellt die Größe des Vierecks die Reparaturkosten dar (je größer, desto höher) und die Farbe des Vierecks nimmt Bezug auf die vorbeugenden Wartungsaktivitäten (je dunkler, desto mehr). Nun gilt es, die großen und auch dunklen Vierecke zu sichten, da an diesen Aggregaten oft vorbeugende Instandhaltung durchgeführt wird, bei gleichzeitig hohen Reparaturkosten. Dies ist auf den ersten Blick ein Widerspruch im Instandhaltungskonzept. Es lohnt sich deshalb, sich darüber Gedanken zu machen, ob hier die Instandhaltungsstrategie ange- passt werden kann, zum Beispiel durch einen anderen Wartungsumfang.

Eine weitere Form der retrospektiven Analyse im Feld des Instandhaltungsmanagements zeigt Bild 4b. Dort wird die Häufigkeit von Wörtern in Instandhaltungsanforderungen am Beispiel von SAP-PM-Meldungen in einer Word-Cloud dargestellt. Die Anzahl an Nennungen hat Auswirkungen auf die Größe der Wörter. Die Farbtiefe als zweite Dimension stellt die Instandhaltungskosten dar, die in Aufträgen, die mit den Wörtern verbunden sind, gebucht wurden. Am Beispiel lässt sich intuitiv erkennen, dass der Betrieb ein größeres Problem mit Undichtigkeiten hat, vermutlich an Abgasleitungen.

\section{NUTZUNG VON MAßZAHLEN IM OPERATIVEN BETRIEB}

Heute werden Prozessanlagen primär mit Hilfe der Basismessgrößen wie Druck, Temperatur, Durchfluss und Füllstand gefahren. Dazu werden Aktualwerte und Vergangenheitsdaten (zeitliche Trends) genutzt. Je aktueller die Werte umso mehr Informationsgehalt kann aus den Daten gezogen werden. Das soll das dargestellte hellblaue Dreieck im linken Teil von Bild 5 symbolisieren.

Wenn Maßzahlen nicht nur retrospektiv sondern bereits im operativen Betrieb genutzt werden, erhöht sich der Informationsgehalt zur effektiven Steuerung von Anlagen. Anlagenfahrer, die Betriebsleitung aber ebenso das IH-Management können mit verdichteter Information arbeiten, die die Zielgrößen einer Fahrweise bereits mehr oder weniger direkt repräsentiert. Der dadurch erhöhte Informationsgehalt und Mehrnutzen 

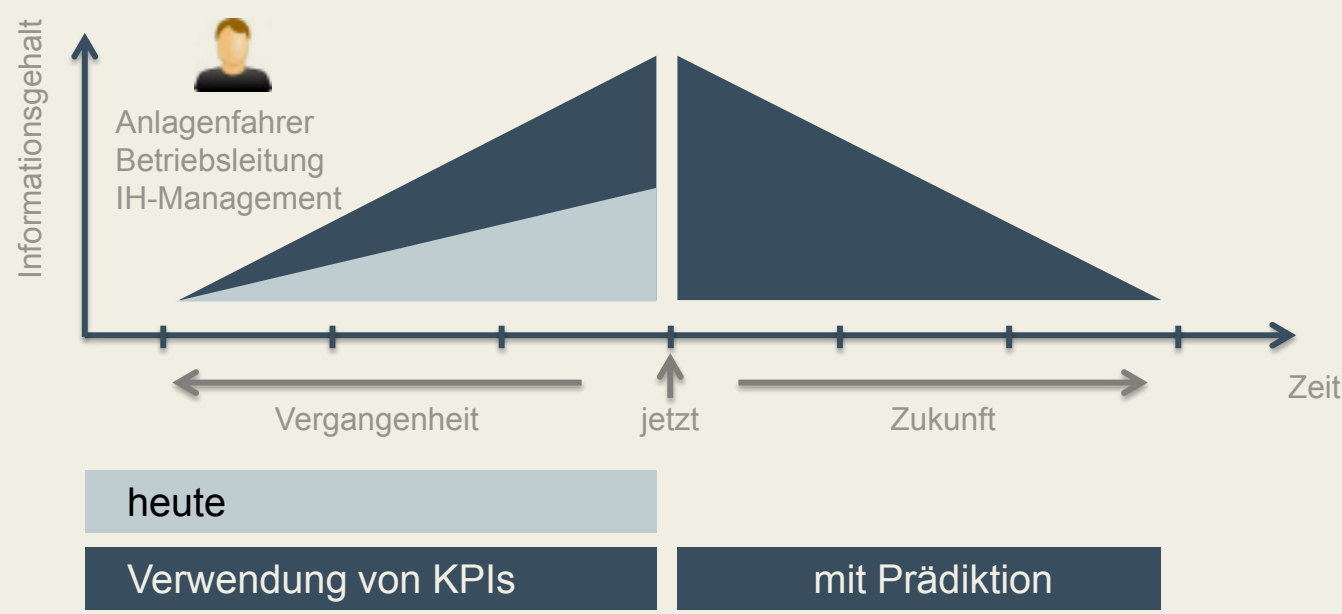

BILD 5: Informationsgehalt von Prozessdaten und Maßzahlen zur Nutzung im operativen Betrieb

werden durch das dunkelblaue Dreieck im linken Teil von Bild 5 dargestellt. Der Nutzen sind zielorientierte Führungsvorgaben, Betriebsassistenz und Vorgaben zur Entscheidungsunterstützung in der Prozessführung. Auch verbesserte Plandaten für das Auftragsmanagement, insbesondere im Rahmen der Feinplanung, können dadurch abgeleitet werden. Systemtechnische Funktionen, die dazu genutzt werden können, sind unter anderem Onlinemonitoring von KPI über Dashboards, KPI-basierte Steuerungen und Regelungen sowie Echtzeitoptimierungen, die sich zum Beispiel für Einsatz- und Ablaufplanungen eignen.

Ein Beispiel für ein Dashboard zum Onlinemonitoring zeigt der Screenshot in Bild 6. Zu sehen sind vier Maßzahlen, die das Betriebspersonal im operativen Betrieb nutzt, um die aktuelle Performance der Anlage

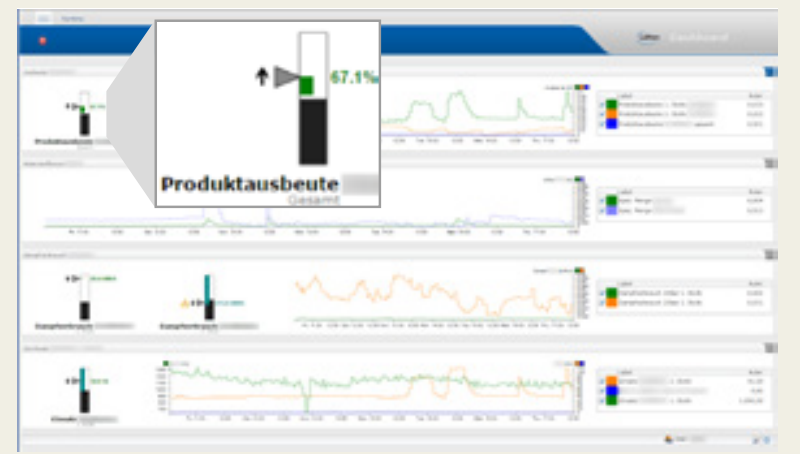

BILD 6: Modernes Dashboard zu bewerten und Änderungen in der Prozessführung zu initiieren. Die Maßzahlen werden zudem in einem Langzeitarchiv gespeichert, um retrospektive Analysen zur Ableitung von mittel- und langfristigen Maßnahmen durchzuführen.

Im Dashboard sind für jede Maßzahl der Aktualwert und ein Kurzzeittrend dargestellt. Interessant ist neben der Darstellung historischer Trendverläufe der Effizienzkennzahlen auch die Nutzung moderner HMI-Elemente. Mit Hilfe von Bullet Charts werden der Messbereich, der Aktualwert, der zuletzt durchlaufende Bereich, Grenzwerte, Warnungen zur Verletzung von Grenzwerten und die aktuelle Tendenz auf einen Blick erfasst.

Ein bedeutender Mehrgewinn wird erzielt, wenn die operative Nutzung von Maßzahlen noch mit modellbasierten Anwendungen kombiniert wird. Mit Hilfe mathematischer Prozessmodelle können unter anderem Vorhersagen für wichtige Prozessgrößen und KPI berechnet werden. Damit steigt die Informationsbasis weiter an. Dieser Mehrgewinn an Information wird in Bild 5 durch das rechte dunkelblaue Dreieck symbolisiert. Auch hier gilt, je näher der Wert am Aktualzeitpunkt ist, umso höher ist der Informationsgehalt. Die Prozessmodelle können auf statischen, dynamischen oder statistischen Methoden beruhen. Oftmals genügen bereits einfache Relationen, um einen ersten Gewinn an Information für die Betriebsmannschaft zu erzielen.

Ein Beispiel hierzu ist eine Anwendung aus dem Kraftwerksbereich, bei dem ein mathematisches Modell des Wasser-Dampf-Kreislaufs dazu genutzt wird, die Performance der Anlage in Hinblick auf den Wirkungsgrad zu erhöhen, siehe auch [16]. In einem Prädiktionsmodus wird das Verhalten einzelner Kenngrößen bei unveränderten Einstellungen 
Prädiktionsmodus

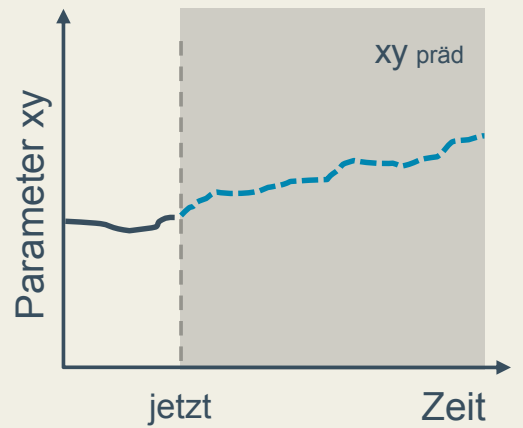

Manövermodus

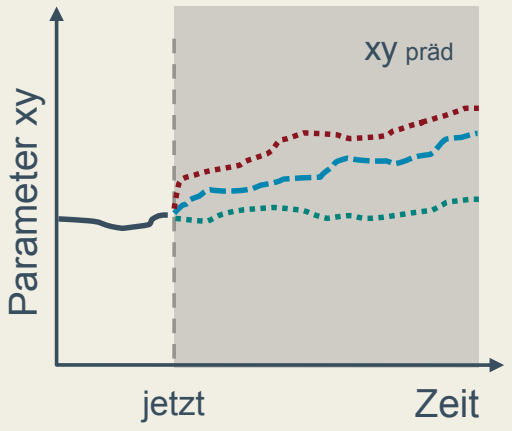

Vorschlagsmodus

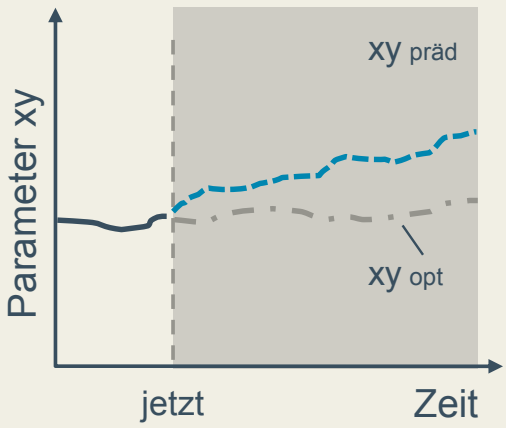

BILD 7: Betriebsassistenzfunktionen auf Basis von Vorhersagen

beziehungsweise Sollwerten vorausgesagt. Dadurch kann der Anlagenfahrer vorzeitig ungünstige Betriebszustände identifizieren und vermeiden. In einem zweiten Schritt kommt ein Manöversimulator zum Einsatz. In diesem Modus kann der Anlagenfahrer während des Betriebs Auswirkungen verschiedener möglicher Fahrweisen oder Sollwertänderungen analysieren. Über einen Vergleich von Ergebnissen kann er sinnvolle Alternativen eigenständig identifizieren und umsetzen. In einem dritten Schritt wird ein Vorschlagsmodus genutzt. Dabei kommt neben dem Modell ein Optimierer zum Einsatz, der mögliche Verstellungen und deren Auswirkungen mit Hilfe eines Gütefunktionals bewertet und eine optimale Betriebsweise vorschlägt.

\section{BEZUGSSYSTEME ZUR BEWERTUNG VON MAßZAHLEN}

Konzeptionell ist es immer wieder eine Herausforderung, geeignete Zielgrößen und Gütefunktionale bei mehreren, gegebenenfalls teilweise in Konflikt stehenden Zielgrößen, auszuwählen und dann Bezugspunkte zur Bewertung der Anlagenperformance zu finden. Die Interpretation und die Freiräume zur operativen Beeinflussung sind dabei abhängig von Randbedingungen, wie dem aktuellen Betriebspunkt und der Anlagenauslastung, der aktuellen Fahrweise und bei Mehrproduktanlagen der aktuell produzierten Produkte, den Rohstoffqualitäten oder externen Randbedingungen, wie zum Beispiel den Umgebungstemperaturen. Es hat sich daher als Vorteil erwiesen, aktuelle Performanceindizes immer bestmöglichen Werten unter vergleichbaren Bedingungen, zum Beispiel im gleichen Lastpunkt, gegenüberzustellen, wodurch sich ein normierter KPI ergibt.
Ein normierter Performanceindex ist das Verhältnis von aktuellem KPI zu einem Referenz-KPI. Dieser Referenz-KPI kann entweder bezogen auf die Vergangenheit der betrachteten Anlage aus der Historie der Datenaufzeichnungen (best demonstrated practice) oder mit Hilfe eines theoretisch errechneten Optimums (best achievable practice) ermittelt werden, siehe Bild 8. Die

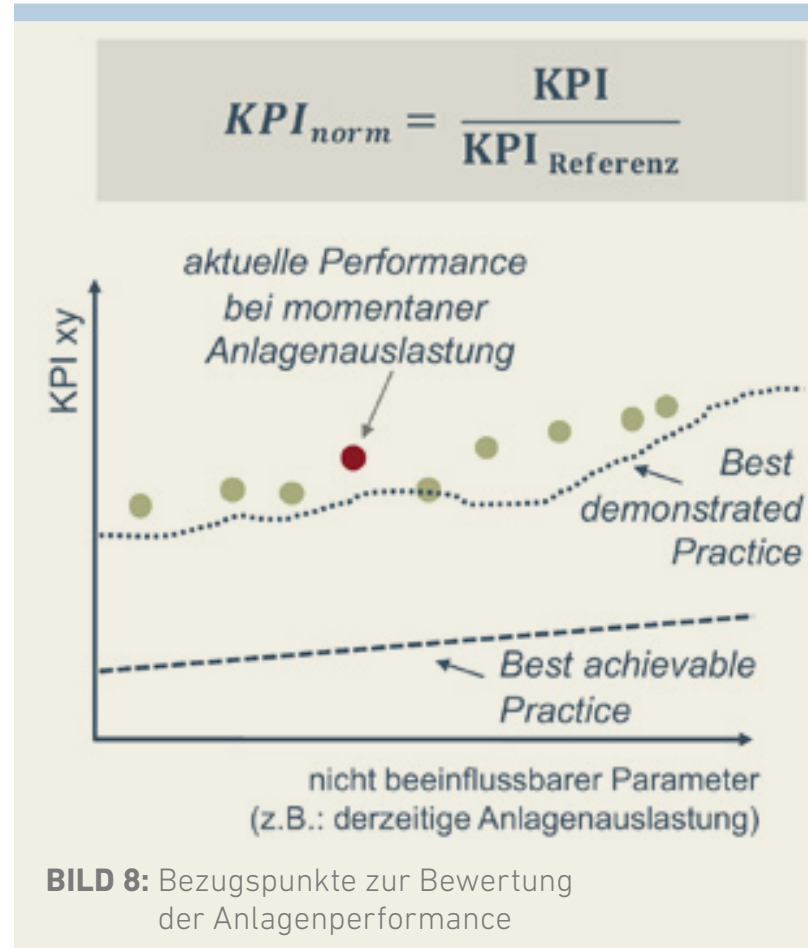




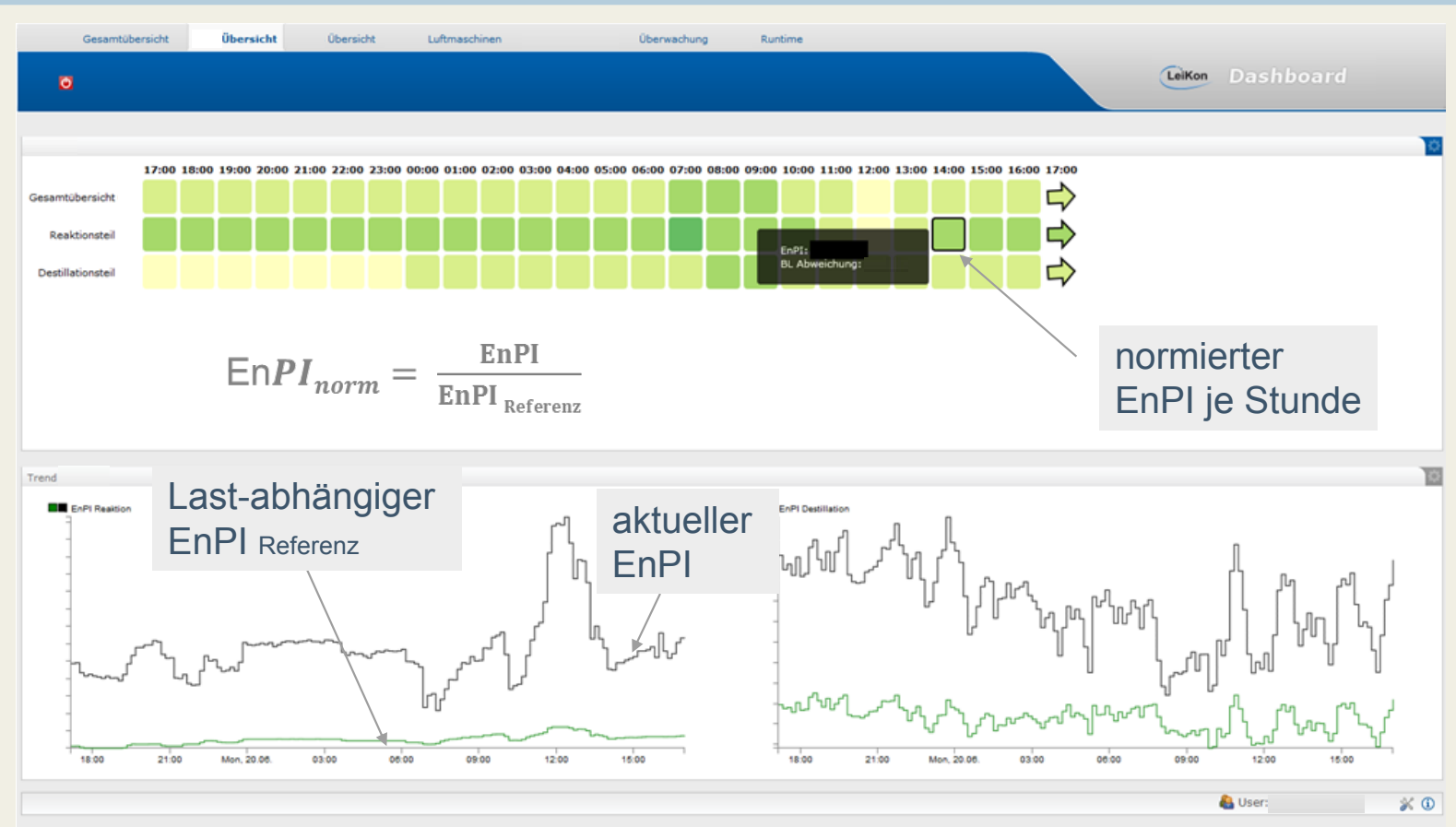

BILD 9: Dashboard mit Darstellungen zur normierten Energieperformance

Praxis zeigt, dass immer, wenn der Mensch Teil der Bewertungskette ist, der Bezug zu real erreichbaren Zielen im Sinne der Best Demonstrated Practice motivierender ist, als der Bezug zu theoretisch möglichen Zielen.

Innerhalb von Dashboards können Anlagenfahrer und Betriebsverantwortliche eine intuitiv deutbare Darstellung des aktuellen Performancezustands gegenüber einem bestmöglichen unter vergleichbaren Produktionsbedingungen nutzen. Ein Beispiel

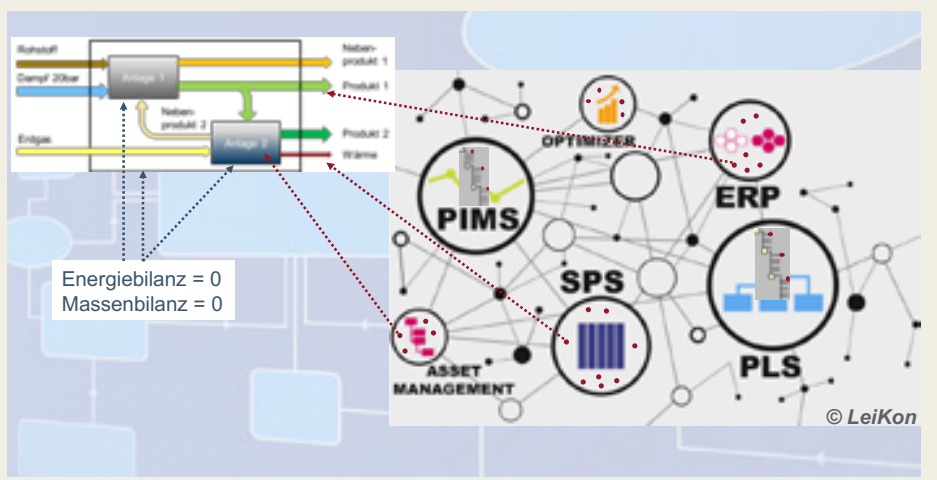

BILD 10: Online nutzbare Informationsmodelle in der Prozessindustrie hierzu ist eine Onlineauswertung, die im Bereich des Energiemanagements genutzt wird, siehe Bild 9. Dargestellt ist die Energieperformance zweier Teilanlagen: der Reaktion und der Destillation. Die unteren Trendkurven zeigen dazu in schwarz den aktuellen Energieperformance-Index (EnPI) und in grün den korrespondierenden Benchmarkwert, der aus der Vergangenheit des Anlagenbetriebs in vergleichbaren Lastsituationen abgeleitet wurde. Der Abstand der Linien zeigt den Anlagenfahrern und Betriebsverantwortlichen intuitiv den aktuellen gegenüber dem bestmöglichen Performancezustand und welches Potenzial zur Performanceverbesserung momentan besteht. Unterstützt wird die Analyse durch eine Kacheldarstellung. Jede Kachel repräsentiert dabei eine Zeitspanne von einer Stunde und zeigt farblich die normierte Performance: je grüner, je performanter. Die Tendenz im aktuellen Stundenraster im Vergleich zum vorherigen Raster wird durch eine Pfeildarstellung am Ende einer Kachelreihe angezeigt.

\section{KONTEXTSENSITIVE BERECHNUNG UND NUTZUNG VON MAßZAHLEN}

Der Hauptnutzen der IT-Technologie der letzten Jahre, insbesondere bezogen auf die Nutzung von Smart Devices, ist die kontextsensitive Nutzung von Daten. Wer benötigt wann, wo, wie und welche Information? Die IT-Unternehmen wie Google, Facebook oder 
Amazon haben dazu mächtige Informationsmodelle geschaffen, die den Kontext der Daten online herstellen und für gezielte Auswertungen nutzen.

Auch in der Prozessindustrie existieren heterogene, vernetzte IT-Systemlandschaften und Kernelemente zur Strukturierung und Zuordnung von Daten, siehe Bild 10. Aber die Information ist heute in Onlinesystemen primär Datenpunkt-orientiert abgelegt. Teilweise werden zwar hierarchische Zusammenhänge aufgebaut, die sich an Anlagenstrukturen orientieren, aber mehr Kontextinformation ist pro Datenpunkt in der Regel nicht hinterlegt. Es werden Informationsmodelle für kontextbasierte Onlineauswertungen benötigt, siehe [17]. Die Informationsmodelle müssen dabei die Bedeutung von Datenpunkten, gegenseitige Abhängigkeiten von Information sowie verfahrenstechnische und anlagentechnische Bezüge und Bilanzräume abbilden können. Die Informationsmodellierung und die Context Awareness sind ein wesentlicher Schlüssel zur effektiven Analyse und Optimierung der Anlagenperformance.

Ein Beispiel dazu ist ein Informationsmodell zur Abbildung von Material- und Energieströmen an einem Standort. Im Rahmen des EU-Forschungsprojektes MORE wurde ein online nutzbares Informationsmodell auf Basis des Werkzeugs IntexcSuite [18] entwickelt. Ein derartiges Informationsmodell enthält Wissen über unterschiedliche Anlagentypen und Typen von Energie- und Masseströmen. Es kennt Flussrichtungen, sodass von einer Durchflussmessung nicht nur der Name der Messstelle und der aktuelle Wert bekannt ist, sondern ebenso, dass der gemessene Durchfluss von Anlage 1 nach Anlage 2 fließt und dabei das Produkt 1 mit dem Energieinhalt y transportiert. Auch Bilanzgrenzen und zugehörige Regeln der Massen- und Energiebilanz sind ein allgemeingültiger Bestandteil des Informationsmodells, siehe Bild 11.

Wenn ein solches Informationsmodell mit den Datenpunkten aus SPS, PLS oder einem Betriebsdatenerfassungs- beziehungsweise Laborinformationssystem verknüpft wird, können ad hoc Onlineauswertungen auf Basis der im Informationsmodell hinterlegten Strukturinformation ausgeführt werden. Der Nutzen liegt in einer verbesserten Echtzeittransparenz der Energie- und Materialströme einer Anlage oder eines gesamten Verbundstandorts, im Aufbau einer zentralen Wissens- und Kalkulationsdrehscheibe und in der leichteren Erkennung von Ursache- und Wirkungszusammenhängen. Die im Informationsmodell hinterlegte Struktur- und Kontextinformation kann direkt zur Berechnung fehlender Messwerte, zur Onlineplausibilisierung von Prozessdaten, zur Onlinekalkulation von Effizienzkennzahlen oder zur Bildung von Adhoc-Bilanzräumen quer zur Anlagenhierarchie genutzt werden. Ein sukzessiver Aufbau eines online nutzbaren Informationsmodells und die Kopplung dieses Ansatzes mit neuartigen Betriebsassistenzfunktionen stellen

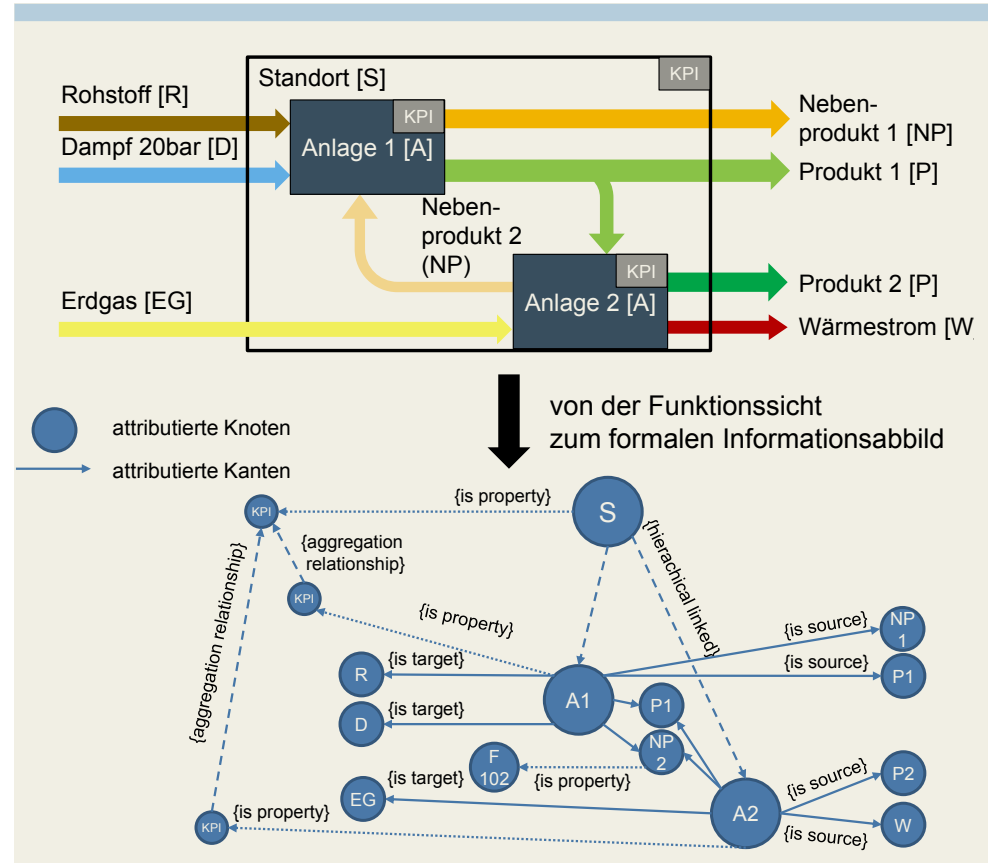

BILD 11: Formale Abbildung einer Funktionssicht

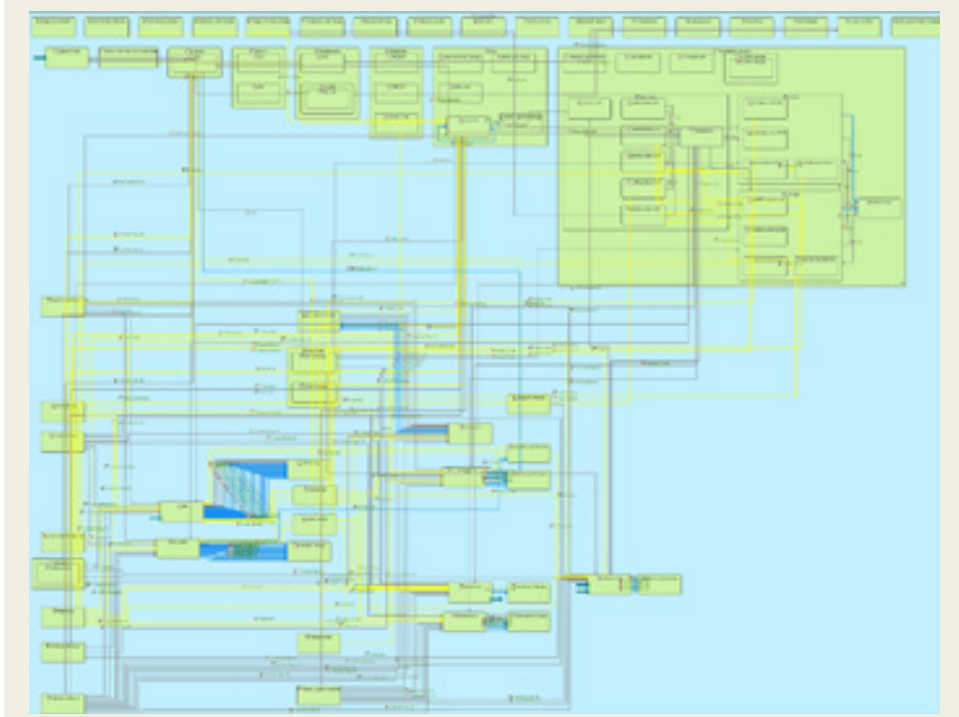

BILD 12: Grafische Repräsentation eines industriell eingesetzten online nutzbaren Informationsmodells der Energie- und Materialflüsse

damit eine neue vielversprechende Herangehensweise dar, um die Anlagenperformance mit Mitteln produktionsnaher IT-Lösungen zu steigern.

Bild 12 zeigt dazu einen Ausschnitt eines automatisch generierten Abbilds einer formalen Beschreibung von 


\section{DANKSAGUNG}

Teile der dargestellten Forschungsergebnisse wurden im Rahmen eines Förderprogramms der Europäischen Kommission unter der Fördernummer 604068 gefördert (FP7 Project MORE - Realtime Monitoring and Optimization of Resource Efficiency in integrated Processing Plants).

Ressourcenflüssen eines Chemiestandorts auf Basis von [18]. Die hierarchischen Anlagenstrukturen, die Anlagenbezeichnungen und wesentliche Energie- und Materialflussverbindungen wurden aus bestehenden Systemen importiert.
Ein Nutzenbeispiel sind mögliche Ad-hoc-Bilanzanalysen quer zur Anlagenhierarchie auf Basis von Typinformation. Eine Frage „Wie ist die Performance meiner Reaktoren in Bezug auf die Energieeffizienz?" ist aus der hinterlegten Information direkt ableitbar. Dabei ist hier ein Reaktor ein spezieller Anlagentyp und die Energieeffizienz ein spezieller Performancetyp. Auf Basis des Informationsmodells können online alle Anlagen vom Typ Reaktor gefiltert werden, deren ein- und ausgehende Energieströme, inklusive der Energieanteile, die in Masseströmen enthalten sind, ermittelt und alle zu einem virtuellen Ad-hoc-Bilanzraum zusammengeführt werden, siehe Bild 13. Durch die Verlinkung der einzelnen Energie- und Massenströme mit Datenpunkten und Historien der bestehenden Leit- und Betriebsdatenerfassungssysteme werden

\section{REFERENZEN}

[1] Mobley, R.K. (1999). Total Plant Performance Management. Gulf Publishing Company

[2] Dhafr, N. and Ahmad, M. (2006). Chemical Plants: Performance Measurement of Processes. Ullmann's Encyclopedia of Industrial Chemistry. Wiley-VCH Verlag

[3] Lindberg, C. F., Tan, S. T., Yan, J. Y., Starfelt, F. (2015). Key performance indicators improve industrial performance. Energy Procedia, 75, S. 1785 - 1790

[4] ISO 50001 (2011). Energy management - Requirements with guidance for use. http://www.iso.org.

[5] ISO 50003 (2014). Energy management systems Requirements for bodies providing audit and certification of energy management systems. http://www.iso.org

[6] ISO 22400 (2014). Automation systems and integration - Key performance indicators (KPIs) for manufacturing operations management. http://www.iso.org

[7] ISO/DIS 20140-5 (2014). Automation systems and integration - Evaluating energy efficiency and other factors of manufacturing systems that influence the environment - Part 5: Environmental influence evaluation data. http://www.iso.org

[8] VDMA 66412 (2010). Manufacturing Execution Systems (MES) Kennzahlen. http://www.vdma.org

[9] VDI 4800 (2016). Blatt 1: Ressourceneffizienz; Methodische Grundlagen, Prinzipien und Strategien. http://www.beuth.de

[10] NE 162 (2017). Ressourceneffizienzindikatoren für die Überwachung und Verbesserung der Ressourceneffizienz in verfahrenstechnischen Anlagen. http://www.namur.net
[11] Real-time Monitoring and Optimization of Resource Efficiency in Integrated Processing Plants. [online] MORE-NMP. Verfügbar unter: http://www.more-nmp.eu

[12] Kalliski, M., Krahé, D., Beisheim, B., Krämer, S. and Engell, S. (2015). Resource efficiency indicators for real-time monitoring and optimization of integrated chemical production plants. Computer Aided Chemical Engineering, 37, S. 1949-1954

[13] Kalliski, M., Beisheim, B., Krahe, D., Engell, S., Krämer, S. and Enste, U. (2016). Real-time resource efficiency Indicators - Material and energy efficient plant operation. atp edition, 58(1), S. 64-71

[14] Kalliski M. and Engell, S. (2016). Real-Time Resource Efficiency Indicators for Monitoring and Optimization of Batch-Processing Plants. The Canadian Journal of Chemical Engineering, 95(2), S. 265-280

[15] Krämer S. and Engell S. (2017) Improving Resource Efficiency in Processing Plants. Wiley-VCH Verlag

[16] Vinnemeier, P., Tomberg, M., Gibbert, M. and Wirsum, M. (2014). Dynamische Modellierung und Simulation des Wasser-/Dampfkreislaufs eines Solarturmkraftwerks für ein Betriebsassistenzsystem. In: Kraftwerkstechnik 2014: Strategien, Anlagentechnik und Betrieb; Beiträge des 46. Kraftwerkstechnischen Kolloquiums am 14. und 15. Oktober 2014 in Dresden, Freiberg: Saxonia, S. 425-442

[17] Kampert D. und Epple U. (2015). Challenges in the Modelling and Operation of Physically Coupled Systems of Systems. In: 8th Vienna International Conference on Mathematical Modelling (MATHMOD 2015), Elsevier

[18] Intexc. [online] Intexc - Integrational Excellence. Verfügbar unter: http://www.intexc.de 


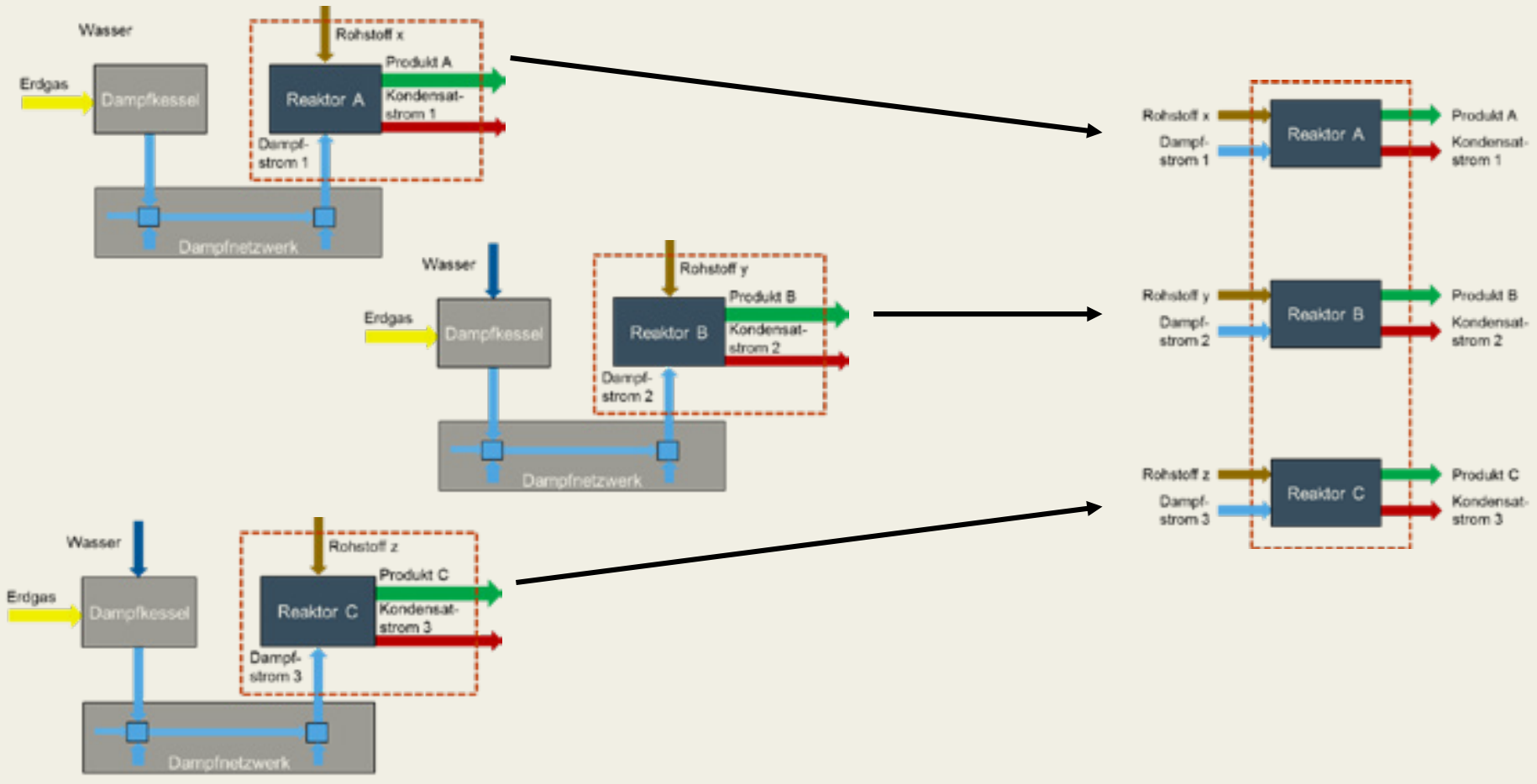

BILD 13: Ad-hoc-Bilanzraum quer zur Anlagenhierarchie gebildet auf Basis von Typinformation

Effizienzkennzahlen auf Basis der hinterlegten Strukturinformation online berechnet.

\section{FAZIT}

Eine möglichst hohe Anlagenperformance ist und bleibt ein wichtiges strategisches Ziel der Anlagenbewirtschaftung in der Prozessindustrie. Je reifer eine Volkswirtschaft und eine Unternehmenskultur sind, umso wichtiger ist es, die bestehenden Ressourcen bestmöglich auszunutzen. Insbesondere kontextbezogene Onlineauswertungen von Daten werden dazu in den nächsten Jahren wertvolle Beiträge leisten, um die Anlagenperformance weiter zu steigern.

MANUSKRIPTEINGANG

06.01.2017

Im Peer-Review-Verfahren begutachtet
AUTOR

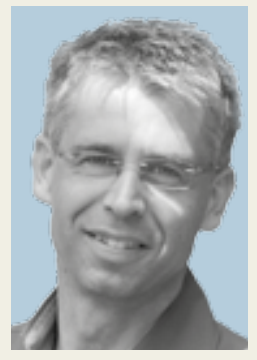

Dr.-Ing. UDO ENSTE (geb. 1968) studierte Elektrotechnik an der RWTH Aachen und hat anschließend dort am Lehrstuhl für Prozessleittechnik promoviert. Er ist seit 2000 Geschäftsführer der LeiKon $\mathrm{GmbH}$, Herzogenrath. Seine Hauptarbeitsfelder liegen im Bereich der operativen Prozessführung, MES sowie funktionaler Sicherheit. Er ist seit 1997 Mitglied im Normenausschuss DIN DKE K931 „Systemaspekte der Leittechnik“ und seit 2011 Obmann des NAMUR-Arbeitskreis 2.4 „MES“.

LeiKon $\mathrm{GmbH}$,

Kaiserstr. 100,

52134 Herzogenrath,

Tel. +49 (0) 240795173 31,

E-Mail: udo.enste@leikon.de 